\title{
Validation of a low field Rheo-NMR instrument and application to shear-induced migration of suspended non-colloidal particles in Couette flow
}

\author{
A.A. Colbourne ${ }^{\mathrm{a}, \mathrm{b}}$, T.W. Blythe ${ }^{\mathrm{a}}$, R. Barua ${ }^{\mathrm{a}}$, S. Lovett ${ }^{\mathrm{b}}$, J. Mitchell ${ }^{\mathrm{b}, *}$, \\ A.J. Sederman ${ }^{\mathrm{a}}$, L.F. Gladden ${ }^{\mathrm{a}}$ \\ ${ }^{a}$ Magnetic Resonance Research Centre, Department of Chemical Engineering and \\ Biotechnology, University of Cambridge, West Cambridge Site, Philippa Fawcett Drive, \\ Cambridge CB3 OAS, UK. \\ ${ }^{b}$ Schlumberger Gould Research, High Cross, Madingley Road, Cambridge CB3 OEL, UK.
}

\begin{abstract}
Nuclear magnetic resonance rheology (Rheo-NMR) is a valuable tool for studying the transport of suspended non-colloidal particles, important in many commercial processes. The Rheo-NMR imaging technique directly and quantitatively measures fluid displacement as a function of radial position. However, the high field magnets typically used in these experiments are unsuitable for the industrial environment and significantly hinder the measurement of shear stress. We introduce a low field Rheo-NMR instrument $\left({ }^{1} \mathrm{H}\right.$ resonance frequency of $10.7 \mathrm{MHz}$ ), which is portable and suitable as a process monitoring tool. This system is applied to the measurement of steady-state velocity profiles of a Newtonian carrier fluid suspending neutrally-buoyant non-colloidal particles at a range of concentrations. The large particle size (diameter $>200 \mu \mathrm{m}$ ) in the system studied requires a wide-gap Couette geometry and the local rheology was expected to be controlled by shear-induced particle migration. The low-field results are validated against high field Rheo-NMR measurements of consistent samples at matched shear rates. Additionally, it is demonstrated that existing models for particle migration fail to adequately describe the solid volume fractions measured in these systems, highlighting the need for improvement. The low field implementation of Rheo-NMR is complementary to shear stress rheology, such that the two techniques could be combined in a single instrument.

Keywords: Rheo-NMR, Low field, Non-colloidal particles, particle migration, Couette flow, Process monitoring
\end{abstract}

*Corresponding author. Tel: +44 1223325426

Email address: JMitchell16@slb.com (J. Mitchell) 


\section{Introduction}

Transport of suspended non-colloidal solid particles is an important process in many industries, including oil and gas production [1] where the controlled placement of a cement slurry [2] and conveyance of solids by drilling fluids [3] are critical for safe and successful operations. Determining the hydrodynamic properties of non-colloidal large particle (diameter $>200 \mu \mathrm{m}$ ) suspensions is a challenge. These complex fluids are not amenable to measurement in narrow-gap rheometric devices due to particle jamming, and violate the narrow-gap approximation [4] in wide-gap geometries such as the cylindrical Taylor-Couette cell [5], where additionally the local rheology may be a function of radius. Nuclear magnetic resonance rheology (Rheo-NMR) measures the spatial variation in velocity and concentration of a sample under shear $[6,7]$ and gives direct access to local shear rate $\dot{\gamma}$ in the wide-gap Couette geometry through the relation

$$
\dot{\gamma}(r)=r \frac{\partial}{\partial r}\left(\frac{v}{r}\right),
$$

where $v$ is the tangential velocity at radius $r$ [8]. In the case of liquids containing suspended non-colloidal particles, the sample evolves over time due to shear-induced radial migration of particles, plus centrifugal radial migration and gravity-driven vertical migration depending on the density mismatch between the solid and liquid components [9-13]. The ability of Rheo-NMR to probe inhomogeneous and opaque liquid systems makes it ideal as a tool for routine process monitoring and quality control in the industrial environment. A commercial Rheo-NMR device is available, designed to fit in the vertical bore of a high field superconducting magnet [14]. However, the installation of high field magnets in a factory or remote location is impractical due to the limitations of cost, safety (strong magnetic fields), and maintenance (requirement for cryogens). Low field permanent magnets, or recently commercialized cryogenfree superconducting magnets, offer lower cost, safer alternatives. Permanent magnet systems also offer the advantage of portability [15]. Low field magnets have been used previously to provide relaxation time measurements of liquid samples in conjunction with shear rheology measurements [16-18]. The usual Rheo-NMR experiment lacks a direct measure of shear stress although custom systems have been proposed that combine NMR and torque sensors [19, 20], albeit still based on superconducting magnets with limited industrial application. Rheo-NMR has been applied to the study of many complex samples, including shear-banding materials [21-23], polymer solutions [24, 25], worm-like micelles [26, 27], liquid crystalline materials [19, 28-31], and colloidal particle suspensions [32, 33]. Shear-induced migration of non-colloidal particles has been studied by NMR imaging in Couette [34-36] and pipe flow [37, 38].

The migration of particles in neutrally-buoyant suspensions in non-uniform shear flows has been described with a variety of models. Diffusive-type models describe the migration process directly as a diffusive process, with particle fluxes directed by gradients in shear rate and particle concentration [39, 40]. Models of the suspension-balance type describe migration as resulting from gradients 
in normal stress of the suspension [41, 42]. In recent years, researchers have been successful in modeling the behavior of dense suspensions using frictional rheology models [43] similar to those previously used for dry granular flow [44]. These can be shown to be equivalent in some cases to suspension balance models [45], but have been generalized successfully to describe additional physics such as suspensions in yield-stress fluids [46] and compaction beyond the jamming limit $[38,45]$.

In this paper we use a low field permanent magnet imaging system to measure velocity and concentration profiles in a wide-gap Couette cell. The hardware and experimental procedures are described in Section 2. Measurements of large non-colloidal particle suspensions in a Newtonian carrier fluid are demonstrated, and the results compared to high field Rheo-NMR acquisitions at matched shear rates in Section 3. For illustration we compare our experimental data to the model of Morris and Boulay [42]. An assumption of this model, as with the other models described above, is that the suspension is locally Newtonian. However, disregarding issues of particle migration, many suspensions exhibit non-Newtonian behavior even at moderate volume fractions and where inertial and Brownian effects are negligible [47]. We observe such effects in our system and subsequently a departure from the predictions of the suspension-balance model.

\section{Experimental}

\subsection{Low field Rheo-NMR}

The low field NMR system comprised a $B_{0}=0.25 \mathrm{~T}$ permanent magnet (Laplacian, UK) of SmCo planar disc poles enclosed in a rectangular iron yoke of dimensions $52 \times 55 \times 48 \mathrm{~cm}$ and approximate mass $150 \mathrm{~kg}$. The field strength corresponded to a resonance frequency of $10.7 \mathrm{MHz}$ for ${ }^{1} \mathrm{H}$. Thermal stability of the magnet was maintained at $28^{\circ} \mathrm{C}$ by active heating and cooling. The vertical bore $\mathrm{rf}$ resonator had an inner diameter of $53 \mathrm{~mm}$ and active damping feedback pre-amplifier (MRF Innovations, UK) for improved response time and signal-tonoise ratio (SNR). The rf power was supplied by a $500 \mathrm{~W}$ CW amplifier (Tomco, Australia). Magnet homogeneity was improved by manual adjustment of an 8 channel shim current supply unit (Resonance Reasearch Inc., USA; model MXD-8). Planar gradient coils driven by high-performance audio amplifiers (AE Techron, USA; model 7224) provided a maximum gradient strength of $g_{\max }=0.125 \mathrm{~T} \mathrm{~m}^{-1}(x$ and $y$ axes $)$ and $g_{\max }=0.14 \mathrm{~T} \mathrm{~m}^{-1}(z$ axis $)$. The water cooled gradient plates maintained the sample temperature at $25^{\circ} \mathrm{C}$. The NMR Experiments were controlled by a DRX-TCP spectrometer (Oxford Instruments, UK) with custom pulse sequences and digital filters [48] optimized for imaging.

Spatially resolved concentration and velocity profiles were obtained using a standard spin-echo imaging sequence [49]. Gaussian rf pulses of duration $t_{\mathrm{p}}=$ $1024 \mu$ s were used to provide a vertical slice of $30 \mathrm{~mm}$ full-width half-maximum (FWHM) and a horizontal slice of $5 \mathrm{~mm}$ FWHM; the rf power was adjusted to provide $\pi / 2$ and $\pi$ pulses as required. For the velocity encoding, three gradient 
increments were used $\left(-g_{\mathrm{v}}, 0,+g_{\mathrm{v}}\right)$ to provide a robust calculation of phase shift. The gradient amplitude was scaled according to the Couette rotation rate to provide the same maximum phase shift in all experiments. The velocityencoding gradient pulses, placed symmetrically about the refocusing rf pulse, had duration $\delta=1.5 \mathrm{~ms}$ and the observation time was $\Delta=11.0 \mathrm{~ms}$. The frequency-encoded read gradient provided a field of view FOV $=59 \mathrm{~mm}$ over 256 pixels, such that the spatial resolution was $\Delta x=229 \mu \mathrm{m}$ with 56 pixels across the Couette gap. The measured profiles extended over the full diameter of the Couette cell, providing antisymmetric velocity components on either side of the rotating center body. The magnitudes of the $y$ velocities were averaged at the absolute $|x|$ coordinates (taking $x=0$ to be the center of the Couette geometry), to provide $\left|\bar{y}_{\mathrm{y}}\right|$ as a function of radius. A recycle time of $500 \mathrm{~ms}$ was used between scans, and 256 repeat scans were summed to improve the SNR, resulting in an acquisition time of approximately 2 minutes per gradient increment.

A custom Rheo-NMR Couette device was constructed for the low field magnet. The outer cylindrical body (stator) was gun-drilled from a rod of polyamideimide (Torlon; Solvay, Belgium) to provide a high-precision machined part. The inner cylindrical body (rotor, or "bob") was machined from polyether ether ketone (PEEK). The Couette geometry had an outer radius of $r_{\mathrm{o}}=20.3 \mathrm{~mm}$ and an inner radius of $r_{\mathrm{i}}=7.5 \mathrm{~mm}$, such that the gap was $r_{\mathrm{o}}-r_{\mathrm{i}}=12.8 \mathrm{~mm}$ with a gap ratio of $\left(r_{\mathrm{o}}-r_{\mathrm{i}}\right) / r_{\mathrm{o}}=0.63$. The total bob length was $252 \mathrm{~mm}$. Rotation of the bob was achieved using a $12 \mathrm{~V}$ d.c. motor with the speed determined by manual adjustment of the voltage supply; an optical encoder in the motor was used to confirm the rotation rate. Rotation rates ranged from $\Omega=3$ to $40 \mathrm{rpm}$; the rotation rate was limited to a maximum of $\Omega=15 \mathrm{rpm}$ when measuring Newtonian liquids due to the formation of Taylor vortexes at higher shear rates.

\subsection{High field Rheo-NMR}

A $B_{0}=7 \mathrm{~T}$ vertical bore superconducting magnet, corresponding to $300 \mathrm{MHz}$ for ${ }^{1} \mathrm{H}$, was used for the high field Rheo-NMR studies. The imaging experiments were controlled by a Bruker DMX300 spectrometer. A bird-cage rf coil of inner diameter $25 \mathrm{~mm}$ was employed. Imaging gradients up to $g_{\max }=0.987 \mathrm{~T} \mathrm{~m}^{-1}$ were available on all three axes. The sample temperature was maintained at $20^{\circ} \mathrm{C}$. A motion-compensated imaging sequence was used with Gaussian rf pulses of duration $t_{\mathrm{p}}=512 \mu \mathrm{s}$, providing a vertical slice of $4 \mathrm{~mm} \mathrm{FWHM} \mathrm{and}$ a horizontal slice of $1 \mathrm{~mm}$ FWHM. A reliable phase shift measurement was achieved with just two gradient increments $\left(0\right.$ and $\left.+g_{\mathrm{v}}\right)$ due to the SNR and stability of the high-field instrument. The velocity-encoding gradient pulses had duration $\delta=1.5 \mathrm{~ms}$ and the observation time was $\Delta=11.8 \mathrm{~ms}$. The frequencyencoded read gradient provided a FOV $=28 \mathrm{~mm}$ over 256 pixels, such that the spatial resolution was $\Delta x=113 \mu \mathrm{m}$, with 53 pixels across the Couette gap. As with the low field data, $\left|\overline{v_{\mathrm{y}}}\right|$ was obtained as a function of radius from the antisymmetric velocity components on either side of the rotating center body. A recycle delay of $6 \mathrm{~s}$ was used and 4 repeat scans were summed, with an acquisition time of less than 1 minute per profile. 
A commercial Rheo-NMR device was used (Magritek, New Zealand and Bruker BioSpin, Germany), designed to fit the vertical bore of the high field magnet. Full details of this device are given in [8]. The radius of the stator $r_{\mathrm{o}}=9.5 \mathrm{~mm}$ and the radius of the rotor (bob) was $r_{\mathrm{i}}=3.5 \mathrm{~mm}$, providing a Couette gap of $r_{\mathrm{o}}-r_{\mathrm{i}}=6.0 \mathrm{~mm}$ and a gap ratio of $\left(r_{\mathrm{o}}-r_{\mathrm{i}}\right) / r_{\mathrm{o}}=0.63$ (consistent with the custom low field Couette geometry). Rotation rates ranged from $\Omega=10$ to $40 \mathrm{rpm}$. Inclusion of a Newtonian marker liquid (water) inside the bob allowed wall slip to be characterized in the high field experiments.

\subsection{Couette geometry correction}

The gap ratio of the two Couette geometries was matched to provide consistent shear rates between the low and high field experiments. In the ideal Rheo-NMR experiment, a thin slice would be selected in the vertical plane so as to be insensitive to the curvature of the inner and outer walls of the Couette cell. Practically, this selected slice must be of finite width and due to the inherently poor SNR available on the low field system it was necessary to select a thick horizontal slice, as detailed in Section 2.1. The relative width of the selected slice compared to the bob diameter is illustrated in Fig. 1(a,b) for the low and high field Couette geometries, respectively. Theoretical $y$-component velocity maps are overlaid on the Couette cell schematics where

$$
\frac{v_{\mathrm{y}}(x, y)}{\Omega r_{\mathrm{i}}}=\frac{x r_{\mathrm{i}}}{r_{\mathrm{o}}^{2}-r_{\mathrm{i}}^{2}}\left(\frac{r_{\mathrm{o}}^{2}}{x^{2}+y^{2}}-1\right),
$$

generalized for a Newtonian liquid. The ideal velocity profiles $v_{\mathrm{y}}(x, 0) /\left(\Omega r_{\mathrm{i}}\right)$ are the solid lines in Fig. 1(c,d) and are independent of Couette geometry due to the matched $r_{\mathrm{i}} / r_{\mathrm{o}}$ ratios. The velocity profiles we expect to measure will differ from these ideal lines due to averaging over the Gaussian-weighted slices. The expected velocity profiles are simulated here by averaging $v_{\mathrm{y}}(x, y)$ over the selected (slice) region; these simulated velocity profiles are given by the dotted lines in Fig. 1(c,d). Comparing the ideal and simulated velocity profiles we see that a significant underestimate of the velocity near the inner wall will be observed in the low field Couette geometry. An equivalent effect occurs at the outer wall but is considered unimportant due to the low velocity in this region. For the high field Couette geometry, a very small difference is observed between the ideal and simulated velocity profiles. A correction based on the simulations, plus an additional term to account for curvature of the streamlines [51], was applied to all measured velocity profiles.

\subsection{Materials}

A Newtonian mixture of water and 2-propanol was prepared in the ratio 2.65: 1 by weight to provide a density of $\rho_{1}=0.96 \mathrm{~g} \mathrm{~cm}^{-3}$. The water was doped with approximately $1.5 \mathrm{~g} \mathrm{dm}^{-3} \mathrm{NiCl}_{2}$ to provide a typical longitudinal relaxation time of $300 \mathrm{~ms}$, permitting a short recycle delay between repeat scans. The nickel salt was chosen for its lower toxicity compared to other standard doping agents. The carrier fluid density was matched to the density of polyethylene 

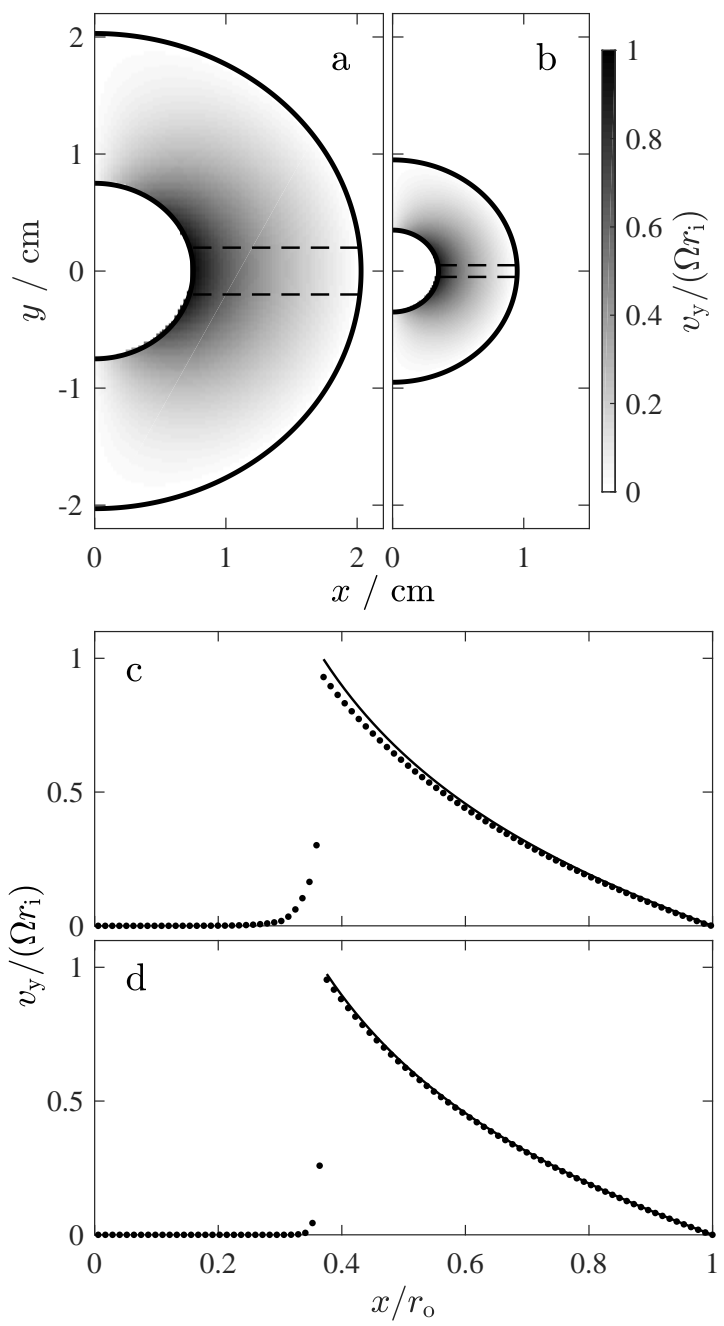

Figure 1: Comparison of Couette geometries in the low and high field magnets. Normalized $y$-component velocity maps are shown for the (a) low field and (b) high field Rheo-NMR geometries; half of the cell is shown in each case where the tangential velocity component is positive (see gray scale). The velocity maps are calculated using eq. (2), valid for a Newtonian liquid. The inner and outer radii of the Couette cells are denoted by the solid black lines. The dashed black lines indicate the FWHM of the $y$-axis Gaussian slice selection. Simulated tangential velocity profiles, calculated by taking a weighted average of velocities over the Gaussian slices, are given by the dotted lines in $(\mathrm{c}, \mathrm{d})$ for the low and high field geometries, respectively. Ideal tangential velocity profiles are given by the solid lines in $(\mathrm{c}, \mathrm{d})$. 
microspheres (Cospheric LLC, USA). These particles were selected for being highly spherical with a narrow diameter distribution in the range 212 to $250 \mu \mathrm{m}$ and were sufficiently large that Brownian motion was neglected. Additionally, we estimated a maximum Reynolds number in our experiments at $40 \mathrm{rpm}$ of $\operatorname{Re} \approx 0.1$, indicating that inertial effects were small (but not necessarily negligible). Particles were added to the liquid to provide solids volume fractions of $\phi=0.2,0.35$, and 0.5 by volume. Precise density matching was achieved by careful adjustment of the water-2-propanol ratio in the carrier fluid at the experimental temperature. During the experiments the Couette cells were sealed to limit evaporation of 2-propanol. Under shear, the particle suspensions were allowed up to $30 \mathrm{~min}$ to reach equilibrium before measurements commenced.

\section{Results and Discussion}

\subsection{Low field validation}

Measured velocity profiles obtained for a Newtonian mixture of water and 2-propanol are shown in Fig. 2(a). The rotation rate of the bob was restricted in the low field instrument due to the formation of Taylor vortexes above approximately $\Omega=15 \mathrm{rpm}$. All the velocity profiles, whether acquired on the low or high field instrument, showed a consistent decrease from the rotating inner wall to zero velocity at the stationary outer wall, as expected. It should be noted that higher tangential velocities are apparent in the low-field instrument due to the larger bob radius. When the tangential velocity component is normalized by the rotation speed of the bob, see Fig. 2(b), all the data collapse to a single smooth line (within experimental uncertainty). These normalized velocity profiles agree with the theoretical prediction of velocity from eq. (2) and demonstrate consistency between the low and high field instruments for a simple Newtonian liquid.

Measured velocity profiles for the polyethylene particles in density-matched liquid are shown in Fig. 3. The bob rotation rates varied between $\Omega=10$ and $40 \mathrm{rpm}$, as indicated by the figure legend. The difference in absolute velocity notwithstanding, the low and high field velocity profiles again collapse to single smooth curves once normalized by the inner wall velocity, see Fig. 3(d-f). The shape of the velocity profile is independent of rotation rate at steady-state but depends on solids volume fraction (SVF). At $\phi=0.2$, the $y$-velocity component decreases with increasing radius, to zero at the stationary outer wall, and is similar to the behavior of a Newtonian liquid. At $\phi=0.35$ the velocity profile is still non-zero but with a higher curvature. At $\phi=0.5$ the velocity decreases to zero at a critical radius $r_{\mathrm{c}} / r_{\mathrm{o}} \approx 0.65<1$, Fig. 3(f). We interpret this region of near-constant $\phi\left(|x|>r_{\mathrm{c}}\right) \approx 0.52$ as jamming where the SVF matches or exceeds its maximum packing fraction, i.e., $\phi \geq \phi_{\mathrm{m}}$, as was found in [10]. This is consistent with the fact that the location of the critical radius does not depend on the rotational speed.

Overall, we consider the consistency of the velocity and particle concentration profiles acquired at low and high field to be indicative of their accuracy, and 


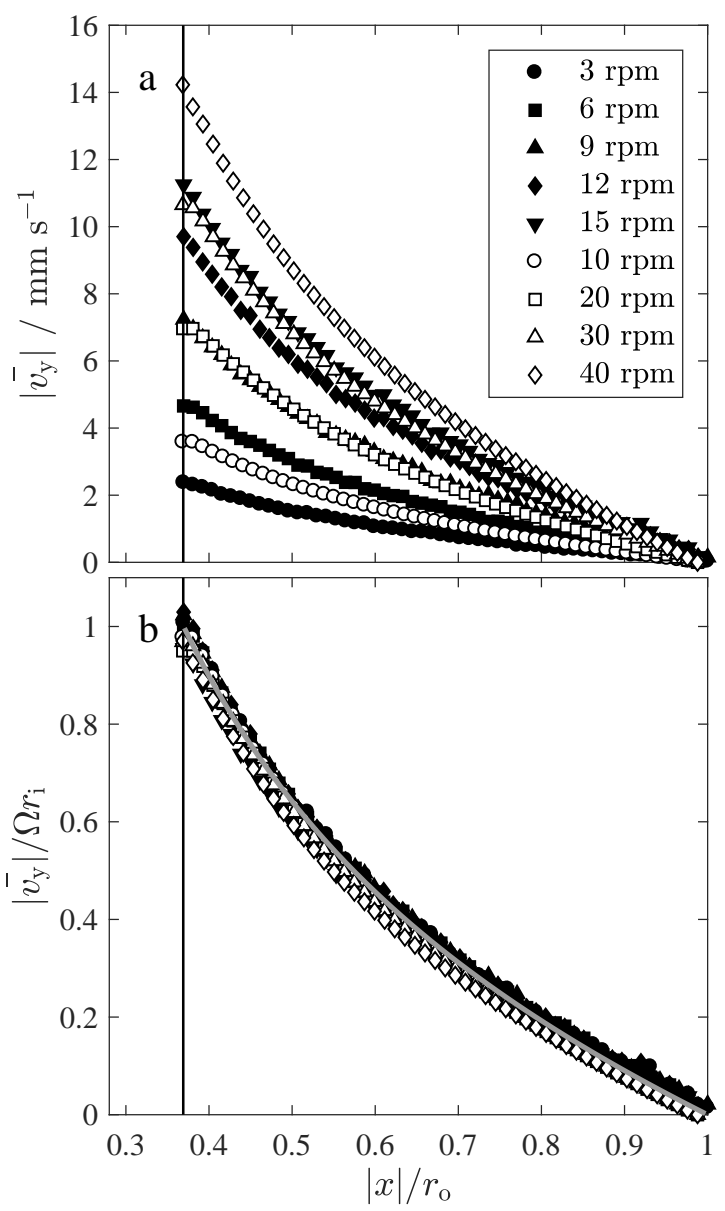

Figure 2: Radial velocity profiles for a Newtonian mixture of water and 2-propanol as (a) absolute velocity and (b) normalized velocity. The filled symbols were obtained at low field, the open symbols at high field. The legend indicates the rotation rate of the inner wall at $r_{\mathrm{i}}$ (position shown by the solid vertical lines) and applies to both plots. In (b) the theoretical normalized velocity profile from eq. 2 is shown by the solid black curve. 
this work demonstrates the feasibility of using low field magnets as quantitative Rheo-NMR instruments.

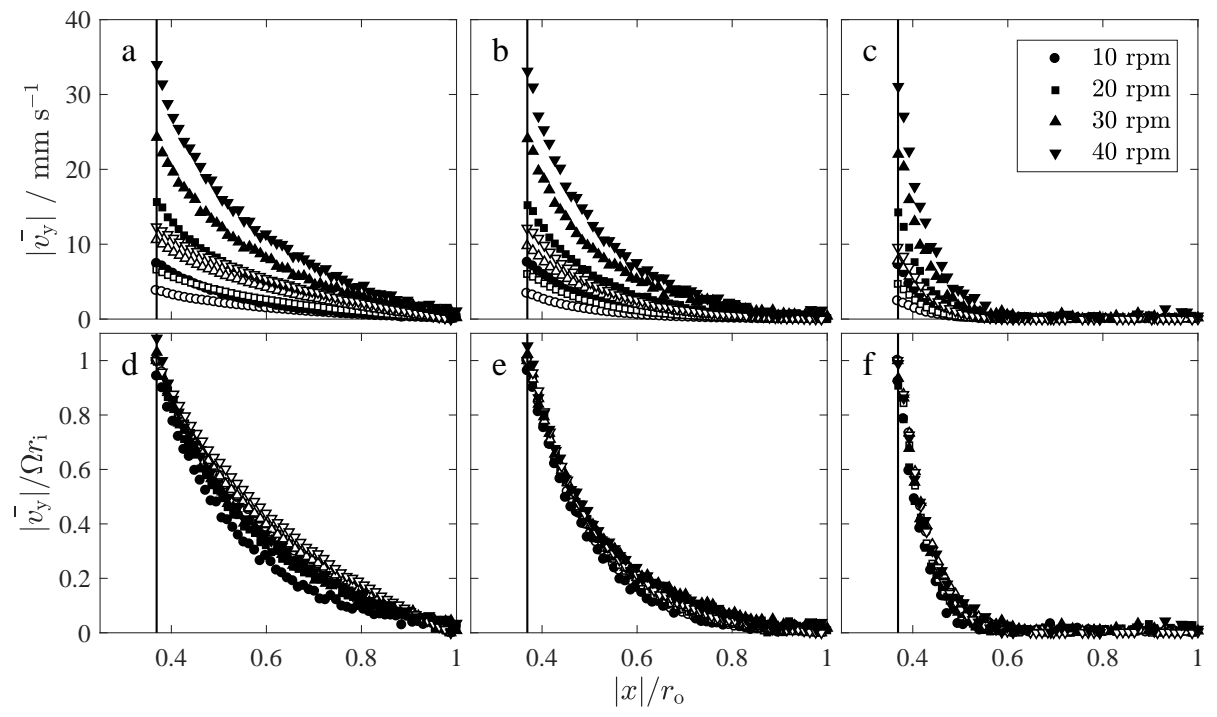

Figure 3: Steady-state radial velocity profiles for a neutrally-buoyant suspension of polyethylene microspheres in a Newtonian carrier liquid as (a-c) absolute velocity and (d-f) normalized velocity. The solid volume fractions were $(\mathrm{a}, \mathrm{d}) \phi=0.2$, (b,e) 0.35 , and $(\mathrm{c}, \mathrm{f}) 0.5$. The filled symbols were obtained at low field, the open symbols at high field. The legend indicates the rotation rate of the inner wall at $r_{\mathrm{i}}$ (position shown by the solid vertical lines) and applies to all plots.

\subsection{Model comparison}

In Fig. 4 we compare the experimental velocity and concentration profiles to the model of Morris and Boulay [42] for steady-state Couette flow. The model describes the radial SVF profile $\phi(r)$, arising from a radial momentum balance, implicitly as

$$
\frac{\eta_{\mathrm{n}}(\phi)}{\eta_{\mathrm{s}}(\phi)}=A_{2} r^{\left(1+\lambda_{2}\right) / \lambda_{2}},
$$

with the relative normal and shear viscosities (made dimensionless with the solvent viscosity) given by

$$
\begin{aligned}
& \eta_{\mathrm{n}}(\phi)=K_{\mathrm{n}} \frac{\left(\phi / \phi_{\mathrm{m}}\right)^{2}}{\left(1-\phi / \phi_{\mathrm{m}}\right)^{2}} \\
& \eta_{\mathrm{s}}(\phi)=1+2.5 \phi_{\mathrm{m}} \frac{\left(\phi / \phi_{\mathrm{m}}\right)}{\left(1-\phi / \phi_{\mathrm{m}}\right)}+K_{\mathrm{s}} \frac{\left(\phi / \phi_{\mathrm{m}}\right)^{2}}{\left(1-\phi / \phi_{\mathrm{m}}\right)^{2}},
\end{aligned}
$$

where $\phi_{\mathrm{m}}=0.52$ is the maximum packing fraction (as found empirically above). $K_{\mathrm{s}}=0.1, K_{\mathrm{n}}=0.75$, and $\lambda_{2}=0.8$ are rheological fitting parameters with the values taken from [42] without modification. Note that eq. (5) matches [50], 
taking account of a typographical error in [42]. For a given value of the constant $A_{2}$, eq. (3) may be solved for $\phi(r) ; A_{2}$ is then fixed by specifying the average SVF. Once the SVF profile is obtained in this way, the shear rate is then given by

$$
\dot{\gamma}(r)=\frac{C}{r^{2} \eta_{\mathrm{s}}(\phi)},
$$

which is integrated to obtain the velocity profile. The wall velocities fix the value of the constant $C$.

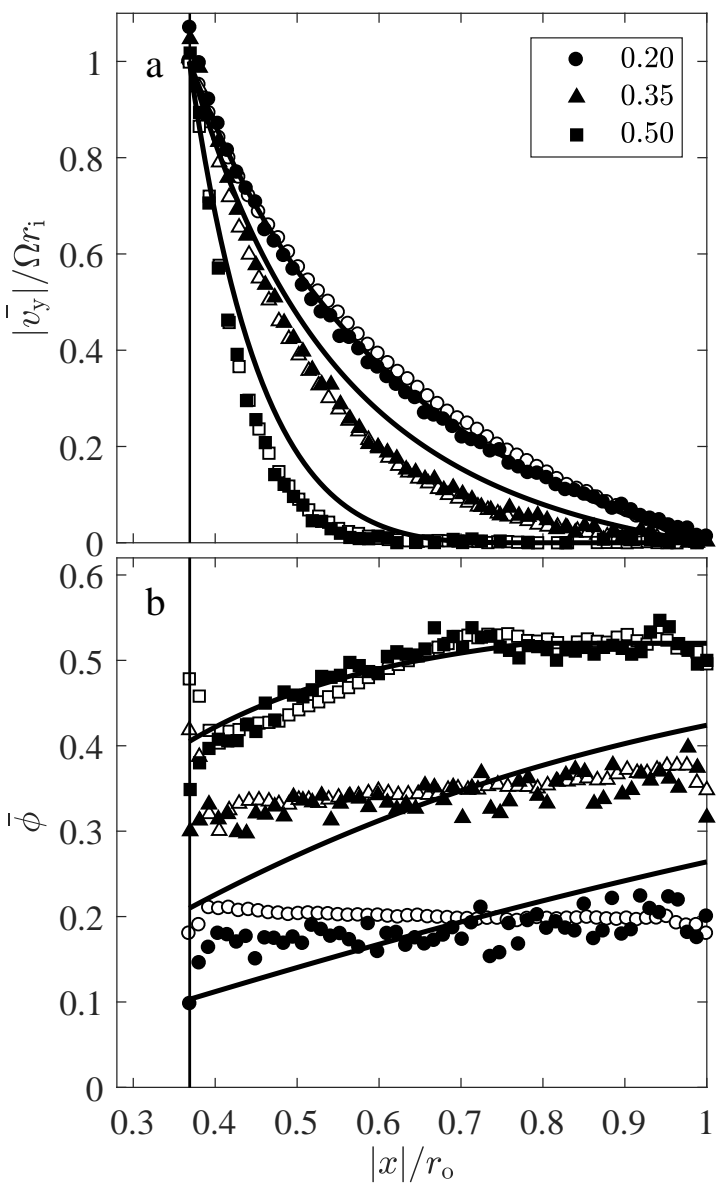

Figure 4: Steady-state radial (a) velocity and (b) SVF profiles for a neutrally-buoyant suspension of polyethylene microspheres in a Newtonian carrier fluid. The position of the inner rotating wall at $r_{\mathrm{i}}$ is shown by the solid vertical lines. The scaled velocities and SVF profiles are independent of rotation rate between $\Omega=10$ to $40 \mathrm{rpm}$, and the data shown are an average over the different rotation rates. The legend indicates $\phi$ and applies to both plots; the filled symbols correspond to the low field experiments and the open symbols correspond to the high field experiments. Predictions from eq. (3) to (6) (solid curved lines) are shown for comparison. 
The velocity profiles predicted by the model match the experiment fairly well, except at higher SVF, see Fig. 4(a). Shortcomings of the suspension-balance model are exposed when we consider the SVF profiles, see Fig. 4(b). The model profile at $\phi=0.5$ shows reasonable agreement, predicting the maximum packing fraction to be reached at a radius $r_{\mathrm{c}} / r_{\mathrm{o}} \approx 0.79$, similar to $r_{\mathrm{c}} / r_{\mathrm{o}} \approx 0.65$ found experimentally. The model makes no prediction beyond this radius and in this region, we have set $\phi=\phi_{\mathrm{m}}$; alternate models exist that include compaction in the jammed region, e.g., [45]. The experimentally-measured SVF profiles for the lower values of $\phi$ do not agree with the model predictions, despite the agreement observed in the velocity profiles. For $\phi=0.35$, the SVF is seen to increase slightly towards $r_{\mathrm{o}}$, but not to the extent predicted by the model; for $\phi=0.2$ the experimental SVF is relatively constant over the Couette gap.

The discrepancies in the observed and predicted SVF in Fig. 4(b) can be explained by strongly non-Newtonian behavior of the suspension, whereas the model for particle migration assumes locally Newtonian suspension rheology. Fitting a power-law model $\tau=k \dot{\gamma}^{m}$ to the experimental velocity profiles, where $\tau$ is shear stress and $m$ the power index, we find $m \approx 0.86$ for $\phi=0.20$, and $m \approx 0.50$ for $\phi=0.35$. It is clear that any model with a locally-Newtonian rheology for the suspension cannot describe these results. In future work we aim to use our low field Rheo-NMR technology to build improved models for particle suspensions in Newtonian and non-Newtonian carrier fluids.

\section{Conclusions}

In this paper we introduced a low field NMR system capable of generating quantitative velocity and concentration profiles in a wide-gap Couette geometry. The instrument was validated against conventional high field Rheo-NMR measurements of a Newtonian liquid and neutrally-buoyant non-colloidal spherical particles in a Newtonian carrier fluid at steady state, at matched shear rates. The two instruments provided consistent velocity profiles for the Newtonian liquid, and consistent velocity and particle concentration profiles for the suspended solids. Experimentally, the particulate system jammed at a SVF of $\phi \approx 0.52$, and appeared to show strongly non-Newtonian behavior (at roughly constant $\phi)$ for the lower volume fractions investigated. This behavior contrasted with model predictions assuming a locally Newtonian system, calling for the design of improved models. The high and low field results remained consistent always, supporting the future use of a low field bench-top instrument for Rheo-NMR studies.

The ability to readily generate robust and quantitative velocity profiles, and hence shear rate profiles, in non-Newtonian liquids using a low field NMR platform has significant advantages, primarily enabling the installation of such a device in the industrial environment. This technology enhances the utility of Rheo-NMR as a process and quality control tool. In future we plan to attach a conventional torque sensor to our Couette cell to provide complementary information from both Rheo-NMR velocity (shear rate) profiles and shear stress rheology. 


\section{Acknowledgements}

AAC thanks the EPSRC (grant EP/K503757/1) and Schlumberger for funding. RB and TWB thank Schlumberger for funding. The authors thank Edmund Fordham, Lukasz Zielinski, Andrew Clarke, and Nate Bachman for helpful discussions. Laurence Hawkes assisted with the sample preparation. John Ashworth assisted with the low field Couette cell design and construction. The $10 \mathrm{MHz}$ magnet was manufactured by Peter Aptaker (Laplacian, UK) with the rf system supplied by Ian Nicholson (MRF Innovations, UK).

\section{References}

[1] G. Maitland, Oil and gas production, Curr. Opin. Colloid Interf. Sci. 5 (2000) 301-311.

[2] A. Doan, A. Brandl, M. Vorderbruggen, D. D. Leonard, Innovative well cementing applications by using large particle sizes of cement additives, SPE 176102-MS (2015) Paper presented at the SPE/IATMI Asia Pacific Oil \& Gas Conference and Exhibition, Nusa Dua, Indonesia, 20-22 October.

[3] J. Li, B. Luft, Overview solids transport study and application in oil-gas industry - theoretical work, Paper presented at the International Petroleum Technology Conference, Kuala Lumpur, Malaysia, 10-12 December IPTC17832-MS (2014) 1-35.

[4] H. A. Barnes, J. F. Hutton, K. Walters, Sections 2.4.4 \& 2.4.5 pp 27-30 in An introduction to rheology, Eds. H. A. Barnes, J. F. Hutton, K. Walters, Vol 3 Rheology Series, Elsevier B.V., Amsterdam, 1989.

[5] G. I. Taylor, Stability of a viscous liquid contained between two rotating cylinders, Phil. Trans. Roy. Soc. Lond. A 223 (1923) 289-343.

[6] P. T. Callaghan, Rheo-NMR: nuclear magnetic resonance and the rheology of complex fluids, Rep. Prog. Phys. 62 (1999) 599-670.

[7] P. T. Callaghan, Rheo-NMR and velocity imaging, Curr. Opin. Colloid Interf. Sci. 11 (2006) 13-18.

[8] K. Hollingsworth, M. Johns, Rheo-nuclear magnetic resonance of emulsion systems, J. Rheol. 48 (2004) 787-803.

[9] D. J. Highgate, R. W. Whorlow, End effects and particle migration effects in concentric cylinder rheometry, Rheol. Acta 8 (1969) 142-151.

[10] G. Ovarlez, F. Bertrand, S. Rodts, Local determination of the constitutive law of a dense suspension of noncolloidal particles through magnetic resonance imaging, J. Rheol. 50 (2006) 259-292. 
[11] G. Ovarlez, S. Rodts, A. Ragouilliaux, P. Coussot, J. Goyon, A. Colin, Wide-gap Couette flows of dense emulsions: Local concentration measurements, and comparison between macroscopic and local constitutive law measurements through magnetic resonance imaging, Phys. Rev. E 78 (2008) 036307.

[12] K. J. Gutam, V. Mehandia, P. R. Nott, Rheometry of granular materials in cylindrical Couette cells: Anomalous stress caused by gravity and shear, Phys. Fluids 25 (2013) 070602.

[13] G. Ovarlez, S. Cohen-Addad, K. Krishan, J. Goyon, P. Coussot, On the existence of a simple yield stress fluid behavior, J. Nonnewtonian Fluid Mech. 193 (2013) 68-79.

[14] P. T. Callaghan, Rheo-NMR: A new window on the rheology of complex fluids, eMagRes 1 (2012) 155-168.

[15] J. Mitchell, L. Gladden, T. Chandrasekera, E. Fordham, Low-field permanent magnets for industrial process and quality control, Prog. Nucl. Magn. Reson. Spectrosc. 76 (2014) 1-60.

[16] V. Räntzsch, K. F. Ratzsch, G. Guthausen, S. Schlabach, M. Wilhelm, Molecular dynamics of polymer composites using rheology and combined rheoNMR on the example of $\mathrm{TiO}_{2}$-filled Poly(n-Alkyl Methacrylates) and Trans-1,4-Polyisoprene, Soft Matter 12 (2014) S4-S13.

[17] V. Räntzsch, M. Wilhelm, G. Guthausen, Hyphenated low-field nmr techniques: combining NMR with NIR, GPC/SEC and rheometry, Magn. Reson. Chem. 54 (2016) 494-501.

[18] V. Räntzsch, M. B. Özen, K. F. Ratzsch, G. Guthausen, M. Wilhelm, Shear rheology and ${ }^{1} \mathrm{H}$ TD-NMR combined to low-field rheoNMR: Set-up and application to quiescent and flow-induced crystallization of polymers, AIP Conference Proceedings 1843 (2017) 040001.

[19] D. Grabowski, C. Schmidt, Simultaneous measurement of shear viscosity and director orientation of a side-chain liquid-crystalline polymer by RheoNMR, Macromolecules 27 (1994) 2632-2634.

[20] J. S. Raynaud, P. Moucheront, J. C. Baudez, F. Bertrand, J. P. Guilbaud, P. Coussot, Direct determination by nuclear magnetic resonance of the thixotropic and yielding behavior of suspensions, J. Rheol. 46 (2002) 709732 .

[21] P. T. Callaghan, Rheo NMR and shear banding, Rheol. Acta 47 (2008) 243-255.

[22] K. W. Feindel, P. T. Callaghan, Anomalous shear banding: multidimensional dynamics under fluctuating slip conditions, Rheol. Acta 49 (2010) 1003-1013. 
[23] S. Kuczera, C. Perge, M.-A. Fardin, T. I. Brox, M. A. K. Williams, S. Manneville, P. Galvosas, Anomalous shear banding revisited with Rheo-NMR and Rheo-USV, Rheol. Acta 54 (2015) 619-636.

[24] D. Bonn, S. Rodts, M. Groenink, S. Rafa, N. Shahidzadeh-Bonn, P. Coussot, Some applications of magnetic resonance imaging in fluid mechanics: Complex flows and complex fluids, Annu. Rev. Fluid Mech. 40 (2008) 209233.

[25] C. J. Davies, A. J. Sederman, C. J. Pipe, G. H. McKinley, L. F. Gladden, M. L. Johns, Rapid measurement of transient velocity evolution using GERVAIS, J. Magn. Reson. 202 (2010) 93-101.

[26] C. Lepper, P. J. B. Edwards, R. Dykstra, M. A. K. Williams, Rheo-NMR studies of a nematic worm-like micelle system in a high-shear-rate regime, Soft Matter 7 (2011) 10291-10298.

[27] T. I. Brox, B. Douglass, P. Galvosas, J. R. Brown, Observations of the influence of Taylor-Couette geometry on the onset of shearbanding in surfactant wormlike micelles, J. Rheol. 60 (2016) 973-982.

[28] H. Siebert, D. A. Grabowski, C. Schmidt, Rheo-NMR study of a non-flowaligning side-chain liquid crystal polymer in nematic solution, Rheol. Acta 36 (1997) 618-627.

[29] C. Lepper, P. J. B. Edwards, E. Schuster, J. R. Brown, R. Dykstra, P. T. Callaghan, M. A. K. Williams, Rheo-NMR studies of the behavior of a nematic liquid crystal in a low-shear-rate regime: The transition from director alignment to reorientation, Phys. Rev. E 82 (2010) 041712.

[30] Y. Geng, P. L. Almeida, G. M. Feio, J. L. Figueirinhas, M. H. Godinho, Water-based cellulose liquid crystal system investigated by rheo-NMR, Macromolecules 46 (2013) 4296-4302.

[31] C. Echeverria, P. L. Almeida, G. Feio, J. L. Figueirinhas, A. D. Rey, M. H. Godinho, Rheo-NMR study of water-based cellulose liquid crystal system at high shear rates, Polymer 65 (2015) 18-25.

[32] J. R. Brown, E. O. Fridjonsson, J. D. Seymour, S. L. Codd, Nuclear magnetic resonance measurement of shear-induced particle migration in Brownian suspensions, Phys. Fluids 21 (2009) 093301.

[33] D. M. Lavenson, E. J. Tozzi, M. J. McCarthy, R. L. Powell, Yield stress of pretreated corn stover suspensions using magnetic resonance imaging, Biotechnol. Bioeng. 108 (2011) 2312-2319.

[34] A. L. Graham, S. A. Altobelli, E. Fukushima, L. A. Mondy, T. S. Stephens, Note: NMR imaging of shearinduced diffusion and structure in concentrated suspensions undergoing couette flow, J. Rheol. 35 (1991) 191-201. 
[35] A. W. Chow, S. W. Sinton, J. H. Iwamiya, T. S. Stephens, Shear-induced particle migration in couette and parallel-plate viscometers: NMR imaging and stress measurements, Phys. Fluids 6 (1994) 2561-2576.

[36] G. Ovarlez, F. Bertrand, P. Coussot, X. Chateau, Shear-induced sedimentation in yield stress fluids, J. Nonnewtonian Fluid Mech. 177-178 (2012) $19-28$.

[37] M. Han, C. Kim, M. Kim, S. Lee, Particle migration in tube flow of suspensions, J. Rheol. 43 (1999) 1157-1174.

[38] S. Oh, Y. Q. Song, D. I. Garagash, B. Lecampion, J. Desroches, Pressuredriven suspension flow near jamming, Phys. Rev. Lett. 114 (2015) 088301.

[39] D. Leighton, A. Acrivos, The shear-induced migration of particles in concentrated suspensions, J. Fluid Mech. 181 (1987) 415-439.

[40] R. J. Phillips, R. C. Armstrong, R. A. Brown, A. L. Graham, J. R. Abbott, A constitutive equation for concentrated suspensions that accounts for shear-induced particle migration, Phys. Fluids A 4 (1992) 30-40.

[41] P. R. Nott, J. F. Brady, Pressure-driven flow of suspensions: simulation and theory, J. Fluid Mech. 275 (1994) 157-199.

[42] J. F. Morris, F. Boulay, Curvilinear flows of noncolloidal suspensions: The role of normal stresses, J. Rheol. 43 (1999) 1213-1237.

[43] F. Boyer, É. Guazzelli, O. Pouliquen, Unifying suspension and granular rheology, Phys. Rev. Lett. 107 (2011) 188301.

[44] P. Jop, Y. Forterre, O. Pouliquen, A constitutive law for dense granular flows, Nature 441 (2006) 727-30.

[45] B. Lecampion, D. I. Garagash, Confined flow of suspensions modelled by a frictional rheology, J. Fluid Mech. 759 (2014) 197-235.

[46] S. Dagois-Bohy, S. Hormozi, E. Guazzelli, O. Pouliquen, Rheology of dense suspensions of non-colloidal spheres in yield-stress fluids, J. Fluid Mech. 776 (2015) R2.

[47] S. Mueller, E. W. Llewellin, H. M. Mader, The rheology of suspensions of solid particles, Proc. Roy. Soc. A 466 (2010) 1201-1228.

[48] A. Valori, J. Mitchell, E. J. Fordham, Digital filter for low-field NMR, Concepts Magn. Reson. 46B (2017) 202-220.

[49] P. Callaghan, Principles of nuclear magnetic resonance microscopy, Oxford University Press, Oxford, 1991.

[50] R. M. Miller, J. F. Morris, Normal stress-driven migration and axial development in pressure-driven flow of concentrated suspensions, J. Nonnewton. Fluid Mech. 135 (2006) 149-165. 
[51] S. Kuczera, P. Galvosas, Advances and artefact suppression in RAREvelocimetry for flow with curved streamlines, J. Magn. Reson. 259 (2015) $135-145$. 